\title{
Nutrition information system (SISFORNUTRIMIL) application With Online Food Record for Indonesia Pregnant Women: Protocol for Randomized Controlled Trial
}

Mira Trisyani Koeryaman ( $\nabla$ mira.koeryaman@port.ac.uk)

Universitas Padjadjaran https://orcid.org/0000-0002-4841-6609

Saseendran Pallikadavath

University of Portsmouth

Isobel Ryder

University of Portsmouth

Ngianga Kandala

University of Portsmouth

\section{Research Article}

Keywords: Eating behaviour, nutrition, pregnancy, system information, SISFORNUTRIMIL

Posted Date: June 17th, 2021

DOl: https://doi.org/10.21203/rs.3.rs-634069/v1

License: (c) (i) This work is licensed under a Creative Commons Attribution 4.0 International License.

Read Full License 


\section{Abstract}

Background: The pregnant women and family may not have the necessary knowledge and skills to estimate nutrient value in food in line with dietary targets and the guidelines, i.e. they do not know whether or not they are consuming the right amount of nutrition needed during pregnancy. The aims of this randomized controlled trial (RCT) is to examine the impact of SISFORNUTRIMIL application on maternal eating behaviour and associated maternal factors such as weight gain, blood pressure, biochemical measurement, and pregnancy outcome.

Methods: The study recruits 112 participants with eligible criteria during December 2019 to January 2020. The allocation of participants is 1:1 to the SISFORNUTRIMIL application user and non-user application, with criteria: singleton pregnancy (22-26 weeks), mother age between 20-35 years), have monthly income and literate. Both groups will receive 12-week standard health services from health professionals and received information about dietary diversity and food record. Particularly, intervention group could be access the SISFORNUTRIMIL on their devices, which provide necessary information about nutrition in pregnancy, food record and food choice includes total serving size calculation. In contrast, the control group only receive food information and food intake record by paper-based, and they should record and calculate the nutrition intake by traditionally.

Discussion: This is the first randomized controlled trial to examine the SISFORNUTIMIL application. This mandate reflects keen interest and motivation for the proposed research study. This is in addition to the researcher's personal efforts to reduce morbidity and mortality rate as well as a general focus on pregnancy complication prevention than nutrition treatment. Thus, an investigation of eating behaviour and associated maternal needed to address this issue. Firstly, the results could extend the scope of knowledge about nutrition intervention to include the Indonesia pregnant women context as well as the Indonesia health ministry context. Secondly, this research could help to change individual health behaviour and activity undertaken by an individual who believes her-self to be healthy.

Trial registration: ISRCTN Registry: ISRCTN42690828. Date of registration: 22 October 2019.

\section{Administrative Information}




\begin{tabular}{|c|c|}
\hline Title $\{1\}$ & $\begin{array}{l}\text { Nutrition information system (SISFORNUTRIMIL) application With Online Food Record for } \\
\text { Indonesia Pregnant Women: Protocol for Randomized Controlled Trial }\end{array}$ \\
\hline $\begin{array}{l}\text { Trial } \\
\text { registration } \\
\{2 \mathrm{a} \quad \text { and } \\
2 \mathrm{~b}\} .\end{array}$ & ISRCTN Registry: ISRCTN42690828. Date of registration: 22 October 2019. \\
\hline $\begin{array}{l}\text { Protocol } \\
\text { version }\{3\}\end{array}$ & $\begin{array}{l}\text { Version } 1.2 \text { approval was obtained from the Universitas Padjadjaran Research Ethics } \\
\text { Committee, with a reference letter number: } 1227 / \text { UN6.KEP/EC/2019 (registration number: } \\
\text { 0619060938). }\end{array}$ \\
\hline Funding $\{4\}$ & Self-funding from the author. \\
\hline $\begin{array}{l}\text { Author } \\
\text { details }\{5 \mathrm{a}\}\end{array}$ & $\begin{array}{l}\text { Mira Trisyani Koeryaman (MTK) as principal author and } \mathrm{PhD} \text { student University of } \\
\text { Portsmouth. } \\
\text { Saseendran Pallikadavath (SP) as principal supervisor } \\
\text { Isobel Ryder (IR) as secondly supervisor } \\
\text { Ngianga Kandala (NK) as third supervisor }\end{array}$ \\
\hline $\begin{array}{l}\text { Name and } \\
\text { contact } \\
\text { information } \\
\text { for the trial } \\
\text { sponsor } \\
\{5 b\}\end{array}$ & Mira Trisyani Koeryaman (mira.trisyani@unpad.ac.id) \\
\hline $\begin{array}{l}\text { Role of } \\
\text { sponsor } \\
\{5 c\}\end{array}$ & $\begin{array}{l}\text { Mira Trisyani (MTK) conceived and design the research project, also was a major contributor } \\
\text { in writing the manuscript. Saseendran Palikadavath (SP) co-writer of the manuscript and } \\
\text { supervised the work Mira Trisyani who guided the entire write-up of the manuscript. Isobel } \\
\text { Ryder (IR) performed the critical revision of the manuscript. Ngianga Kandala (NK) } \\
\text { determined the data analysis for this study. }\end{array}$ \\
\hline
\end{tabular}

\section{Introduction}


Since 2004, the World Health Organization (WHO) tried to achieve the challenges of the global strategy on a diet, physical activity and health that focused on maternal health and nutrition before and during pregnancy. As such, women need to set the optimal birth outcomes through good nutrition practice during pregnancy (Symington, 2018). Proper nutrition during pregnancy is necessary for mother and infant outcomes and indeed for later life (Scott, 2007). However, for pregnant women, healthy diet and dietary intake are often insufficient to meet these needs; there likely are multiple nutritional deficiencies, particularly in low and middle-income countries (WHO, 2016). South-East Asia region is the one of the regions has a contribution in highly prevalent of maternal under nutrition (WHO, 2016). Lack nutritional food during pregnancy, unattended prenatal care, belief taboos that prevent uptake of nutritious food during pregnancy, and binge eating, are more likely to be likely associated with maternal morbidity and mortality prevalence (Guggino, 2016). Some key driving factors, such as cultural practices, poverty and weaknesses in nutrition governance, can contribute to maternal and child under nutrition (Indonesia Nutrition profile, 2014; Schumer, 2014; Nguyen, 2017).

According to the nutrition profile in Indonesia, approximately 9.2 million (37\%) from 24.5 million children under five years of age are stunted, and $24-27 \%$ in some regions is low birth weight. Indonesia is one of the countries with a large contributor to maternal mortality rate (MMR) in the world, particularly in SouthEast Asia (WHO, 2016). Maternal morbidity in Indonesia mainly occurs during labour due to other reasons $53.4 \%$ including hypertension, prematurity, the breech position of the foetus and swelling. The secondhighest morbidity during pregnancy was bleeding $25.0 \%$, premature rupture of membrane $21.1 \%$, and convulsion $2.3 \%$ (Widyaningsih et al. 2017). While, the neonatal mortality caused by prematurity $35.5 \%$, birth asphyxia and trauma $21.6 \%$, congenital anomalies $17.1 \%$, sepsis $13 \%$, and other $5.4 \%$ (Unicef, 2015). These could profoundly be influenced by inadequate nutrition during pregnancy. The prevalence of imbalance of nutrition requirement has significantly increased in developed countries over recent decades, with an estimated $52 \%$ to get anaemia high-risk and $25.8 \%$ to have diabetes (WHO, 2014; Yuan, 2016). In particular, at present, the stunting prevalence rate in West Java is 29.2 per cent in 2017, identified as being caused by maternal factors (Beal et.al 2017).

The current study is motivated by a personal desire to tackle this growing problem, derived from Indonesia's nutrition profile and personal experience as a researcher and thesis supervisor in nutrition pregnancy topics. Despite, extensive research in this field, there is no effectiveness solutions/intervention currently available to address this problem (Kavle, 2017). The insufficiency intervention due to lack measurement of maternal dietary consumption and suboptimal counselling by health provided services focused on maternal diet and weight gain (Lucas 2014 in Arrish, 2017). Therefore, to the author's knowledge, this study proposes solutions to the absence of integrated individual interventions between information systems and food record; the authors devised a group-based intervention in an effort to develop a self-monitoring dietary assessment method.

\section{Objectives $\{7\}$}

Research objectives: 
Primary objectives:

- To determine if maternal eating behaviour effects of user SISFORNUTRIMIL application after three months were at standard criteria of Indonesia pregnant women, compare with non-users

Secondary objectives:

- To determine if clinical and biochemical assessment effects after three months were at an average level, compare with non-user SISFORNUTRIMIL application.

- To identify the effect of SISFORNUTRIMIL application toward neonatal birth weight, compare with non-user SISFORNUTRIMIL application.

- To compare intra-participant effects in primary and secondary objectives for determining whether individual changes were sustained at three months from baseline assessment.

\section{Hypotheses}

- The null hypothesis $(\mathrm{Ho})$ is that there is no difference in maternal eating behaviour including related factors such as maternal weight, blood pressure, haemoglobin levels, blood glucose levels and neonatal birth weight between SISFORNUTRIMIL application user (intervention) and non-user (control) groups.

- Alternative hypothesis $(\mathrm{Hi})$ was that a difference existed between the two groups.

\section{Trial design $\{8\}$}

This study will use randomized control trial (RCTs) method to attain the study objectives. In order to avoid selection bias in determine of maternal eating behaviour and associated maternal assessment, the theory of change and randomization technique should be considered (Rogers, 2018). The framework model of this study, which sets out the theory of change for nutrition intervention. All eligible participants will be selected by matched pair randomization, which similarity of maternal age, gestational age, income, education level, and parity. To allocate between intervention and control groups, the researcher will use free online randomizer "QuickCalcs by GraphPad (White et al., 2018).

The guideline intervention referring to Standard Protocol Items (SPIRIT) checklist 2013 version and Consolidated Standards of Research Trials (CONSORT) items-in particular for non-pharmacologic treatment (Chan, et al, 2013; Boutron, 2008), and conjunction with CONSORT 2010 as the latest version (Schultz et al, 2010). This protocol describes a two-armed randomised control trial (RCTs) during 12 weeks, to determine maternal eating behaviour and associated maternal. The recruitment of participants will be screened to use inclusion and exclusion criteria. Firstly, the women will be recruited into four strata: as a permanent patient of PUSKESMAS or private clinic, the age between 20 to 35 years, gestational age 22-26 weeks and a singleton pregnancy, and necessary ability use any devices. Then, informed consent will be obtained prior to any study-related procedures being performed, including the 
discontinuation of the current study. Blood samples (haemoglobin and blood glucose) will be drawn to confirm eligibility based on clinical laboratory parameter.

\section{Methods}

\section{Participants, interventions and outcomes}

\section{Study setting $\{9\}$}

The data will be collected at seven sites of public health center (PUSKESMAS) of Bandung city, West Java, Indonesia, which five Puskesmas completed by a basic emergency obstetric and new-born care (BEmONC) room.

\section{Eligibility criteria $\{10\}$}

The population of this study were all pregnant women of restricted age at between 19-30 years and gestational age between 22-26 weeks who were outpatients in the maternal and child health clinic of PUSKESMAS or private clinic and received regular antenatal care. The following inclusion and exclusion criteria were applied to select participants in the trial.

\section{Inclusion criteria}

1. Permanent patient of Puskesmas or clinic

2. Age between 19-30 years old,

3. Primigravida and singleton pregnancy,

4. Gestational age $22-26$ weeks

5. Educated

6. Have monthly income

7. Women who are able to use any electronic devices

\section{Exclusion criteria}

1. Pregnant women with serious medical condition such as food allergy, bulimia and chronic illness

2. Diagnosed with a mental illness patient,

3. Non-permanent patients of PUSKESMAS,

4. Do not have any electronic devices, and

5. Participants who fall in the inclusion criteria but do not give or lack the capacity to give consent.

\section{Who will take informed consent? $\{26 a\}$}

All women who eligible in criteria will be asking to read the information sheet and ask them to participate in the study. A participants' information sheet translated into the local language, had detailing all the 
information about the study covering objectives, length period, outcomes and post study. The participants will have adequate time to read and understand the information sheet before committing to the study (min 72 hours). Participants will also be given the opportunity to ask the researcher any questions they may have at any time before and during the interview.

\section{Additional consent provisions for collection and use of participant data and biological specimens $\{26 \mathrm{~b}\}$}

Informed consent will be obtained prior to any study related procedures being performed, including blood samples.

\section{Interventions}

\section{Explanation for the choice of comparators $\{6 \mathrm{~b}\}$}

To allocate between intervention and control groups the researcher will use free online randomizer "QuickCalcs by GraphPad allow a researcher to specify the number of participants and number of groups, and quickly return a list showing which participant go to which groups (White at al., 2018). Before participants were randomized, they will be identified by matching pair criteria, which helps to ensure balance and reduces the required sample size (White et al., 2018). The matched pair criteria determined by two groups' similarity such as maternal age based on age range (20 to 30 years), gestational age (2226 weeks), parity (primipara), education level (secondary and higher education), and social-economic status represented by monthly household income (under and over on minimum wages in West Java). The principal researcher notified all pregnant women that they had the opportunity to access the SISFORNUTRIMIL application; however, the randomizer will choose one of them.

\section{Intervention description $\{11 \mathrm{a}\}$}

\section{Intervention group}

The participants who recruited into intervention group will be provided to open the SISFORNUTRIMIL application through http://www.sisfornutrimil.com. This website address can be access by pregnant women, which is automatically matched with the screen size of the device used such as computer or notebook, and tablet or smartphone. The application feature includes the form of food choice as nutrition intake suggestion, food record or food log history. The food choice form shows the suggestion of nutritional intake that could be consumed by pregnant women at each mealtime. The information provided will vary every day; the application will display the nutritional value of each recommended food and the total nutrition obtained when all of the recommended food is consumed. The references data were based on dietary recommendations for Indonesian population and those published by the health ministry of Republic Indonesia. Energy estimates for each food and drink were calculated based on food groups and subgroups. Standard measuring equipment on common various size containers (e.g. plates, cups, bowls, spoons and glasses) used to assist in quantifying portion sizes. There are 321 types of food, which are divided into 7 main food source groups including carbohydrates, protein, vegetables, fruits, 
light meals, fast food, and beverages. The food record or food log history page will be able to record all food consumed within one day.

\section{Comparator group}

The comparator is a control group who does not have access to SISFORNUTRIMIL application only received standard prenatal care. The data will be carried out in baseline and every antenatal care visits after baseline. Participants in the control arm will receive standard prenatal care at maternal and child health clinic, PUSKESMAS from health professionals, plus souvenirs which have been prepared as gifts (such as hand towel/meals box). In general, the standard prenatal care and tests may include checking blood pressure and weight; baby's heart rate; measuring women abdomen; measurement of haemoglobin levels, blood glucose. The group control will be asked to read information about nutrition in pregnancy through leaflets that have been given by researcher, and also they received an explanation about how to fill in the food record form during 12 weeks.

Then both of groups will be measured of birth weight on the day of birth. In addition, the women who have completed food records online or offline, they will be allowed to get transportation fees. Later, the participants will be entered into a draw to win a gift equal with $£ 10$ or 200.000 IDR, if they are able to complete the intervention.

\section{Criteria for discontinuing or modifying allocated interventions $\{11 \mathrm{~b}\}$}

The study may be discontinued at any time by the research committee, or other government agencies as part of their duties to ensure that research participants are protected. There is no prohibits intervention, however the researcher will remove the women from this study if participant do not have a completely data such as missed of baseline and after baseline measurement, moved out cities, and miscarriages or Intra Uterine Foetal Death (IUFD) between periods on access the SISFORNUTRIMIL application (in 12 weeks' intervention periods).

\section{Strategies to improve adherence to interventions $\{11 \mathrm{c}\}$}

The data collector will send text message every week end to reminder all participant input their food intake record. The evaluation of intervention process including laboratory test will be monitored in antenatal care schedule by data collector.

\section{Relevant concomitant care permitted or prohibited during the trial $\{11 \mathrm{~d}\}$}

There are not concomitant interventions and harmful action. The women will receive standard antenatal care from health professional including laboratories test as standard assessment. The researcher will remind the woman to record the food via telephone at least once a week and ensure they ensure they have no difficulty accessing and using the App.

\section{Provisions for post-trial care $\{30\}$}


There is no ancillary and post-trial care. However, the principal investigator will give the transportation fees as compensation for those who have agreed to have a laboratory test and a souvenir as a gift for completing all interventions.

\section{Outcomes $\{12\}$}

The primary outcome will identify of women's eating behavior include diet quality such as dietary diversity, food intake and weight gain during pregnancy. The secondary outcomes will identify the optimal pregnancy experience at twelve weeks after baseline measurement such as biomarkers test, and birth weight measurement at the end of pregnancy.

\section{Maternal eating behaviour measurement}

Eating behaviour will be measured by Minimum Dietary Diversity for Women of reproductive age (MDDW) for women aged 15-49 years who have consumed at least five out of ten defined food groups in the previous day or night (Martin-Prevel, et al (2015) in FAO, 2016). The MDD-W to describe one important dimension of women's diet quality include micronutrient adequacy, summarized across 11 micronutrients. The food diversity indicators can be measured using list-based method (FAO, 2016). Other measure will be assessed by Adult Eating Behaviour Questionnaire (ABEQ) (Hunot, 2016). The ABEQ score might inform an individual at risk of experiencing inappropriate weight gain. The eating behaviour measured define into eight appetitive traits: Hunger $(H)$, Food Responsiveness (FR), Emotional OverEating (EOA), Enjoyment of food (EF), Satiety Responsiveness (SR), Emotional Under-eating (EUE), Food Fussiness (FF) and Slowness in Eating (SE). Each item responses were recorded on a 5-point Likert scale ranging from 'Strongly Agree' to 'Strongly Disagree'. Mean scores were calculated for each subscale. Furthermore, to determine the level of respondents' achievement was categorized into five criteria "excellent" (85\%-100\%), "Good " (66\%-84\%) , " Average " (51\%-65\%), " Fair " (36\%-50\%), and "Poor"(0-35\%).

Gestational weight gain measurements can be determined by weight, height, and gestational age (Committee, O. N. S. D. P., \& Institute, O. M., 1990). Measurement of weight $(\mathrm{kg})$ and height ( $\mathrm{cm})$ is determined based on the nearest $0.05 \mathrm{~kg}$ for weight, and $0.1 \mathrm{~cm}$ for height (Symington, 2018). Detailed description of the standard operating procedure (SOP) for weight and height measurement is providing in appendices. The indicators of maternal weight gain are divided into 3 categories: less, normal and excess than total weight gain in each trimester (Scott. 2007). Whereas, the rate of weight gains according to Committee, O. N. S. D. P., \& Institute, O. M., (1990) divide at different stage of pregnancy, as follow: 13 to 20 weeks usually about 0.15 to $0.69 \mathrm{~kg}(0.3$ to $1.5 \mathrm{lb})$ per week; 20 to 30 weeks about 0.31 to $0.65 \mathrm{~kg}(0.7$ to $1.4 \mathrm{lb})$; and 30 to 36 weeks about 0.18 to $0.61 \mathrm{~kg}(0.4$ to $1.3 \mathrm{lb})$ per week.

Food intake and food pattern will be assessed by food record. The food intake to estimation and classify subject correctly according to a usual energy and nutrient intake (Pereira, 2010). The criteria of sufficient nutrient per kilocalorie of energy intake categorized divided into 4 groups according to Institute of Medicine (2005): Good (>80\% RDA), modest (70-79\% RDA), poor (60-69\% RDA), and deficit ( $<60 \%$ RDA). Food frequency questionnaire (Shim, 2014), are commonly assumed to provide accurate estimates of 
habitual energy intake. Three days food records will selected as the method for assessing dietary intake in the RCT. Pregnant women will asked to maintain a 3-day (2 days in work day and 1 day in week end) record all the foods and drinks they The application will be stored data in MySQL database as structure query language in Entity Relationships Diagram form (ERD).

\section{Blood pressure and biochemical measurement}

Blood pressure will be assessed for gestational hypertension prevention (Grosvenor, 2006). Blood pressure is measured at each prenatal visit and using calibrated equipment according to international guidelines $(\mathrm{mmHg}$ ) (Weber, 2014). Women blood pressure will be measure from the right arm using a standard mercury sphygmomanometer after 5 min of rest with the subject in the sitting position. Hypertension was defined as systolic blood pressure $\geq 140 \mathrm{mmHg}$ or diastolic blood pressure $\geq 90$ $\mathrm{mmHg}$ by using 2007 European Society of Hypertension (ESH)-European Society of Cardiology (ESC) Guidelines for the management of arterial hypertension (Mansia et al., 2007 cited by Yuan 2016).

Pregnancy experience assessment will be measured using blood sample test. It screens pregnancy complication includes anaemia and gestational diabetes mellitus (GDM). Standard procedures are used for biochemical analyses such as haemoglobin $(\mathrm{Hb})$ and blood glucose test (Symington, 2018). According to the World Health Organization, anaemia is diagnosed when a blood test shows of haemoglobin level less than $110 \mathrm{~g} / \mathrm{L}$ in pregnant women (Wang, 2018). Additionally, the GDM diagnostic criteria for non-fasting glucose test followed the World Health Organization (1999) and America Diabetes Association (2003) are categories non-diabetes mellitus ( $<140 \mathrm{mg} / \mathrm{dL}$ ), impaired glucose tolerance (140 $\mathrm{mg} / \mathrm{dL}-<200 \mathrm{mg} / \mathrm{dL}$ ), and diabetes mellitus (>200mg/dL).

\section{Pregnancy outcomes measurement}

Pregnancy outcomes measurement will be take in the end of pregnancy. The infant is weighted on a calibrated scale with minimum of clothing, namely only a vest, and without nappy, and recorded to the nearest $5 \mathrm{~g}$ (Symington, 2018). The categories neonatal birth weight measured according to Chan, et al (2011) are dividing into three groups: very low birth weight $(<1.500 \mathrm{~g})$, low birth weight $(<2.500 \mathrm{~g})$, and normal birth weight (2.500 or more).

\section{Participant timeline $\{13\}$}

Baseline Assessment: One week after screening procedure, the participant will be performed to fill the participants provided standardized of characteristic information on 25 items to describe the socioeconomic status of the Indonesia population. At the time of baseline measurements, maternal diet quality will be assessed by the MDD-W and ABEQ.

Post-intervention: Following the completion of 12-weeks intervention, participants will complete a postintervention, food record online and 7-Day food record. Besides, completed the post-intervention measurement includes Adult Eating Behaviour Questionnaire' (AEBQ), gestational weight gain, blood 
pressure and biochemical assessment. See table 1 for a list of measures used in the post-intervention survey.

Follow-up: At the end of pregnancy, all participants will complete follow-up data. The neonatal birth weight assessment will be collected. See table 1 for a list of measures used in the follow-up survey.

Table 1 List of Study Measurement

\begin{tabular}{|c|c|c|c|c|c|}
\hline \multirow{3}{*}{ Study Measurement } & \multirow{3}{*}{$\begin{array}{l}\text { Methods of sample } \\
\text { used }\end{array}$} & \multicolumn{4}{|c|}{$\begin{array}{l}\text { Study Phases } \\
\end{array}$} \\
\hline & & Screening & $\begin{array}{c}\text { Baseline } \\
\text { Assessment }\end{array}$ & Intervention & Follow-up \\
\hline & & & $\begin{array}{c}1 \text { week } \\
\text { after } \\
\text { screening }\end{array}$ & $\begin{array}{l}12 \text { weeks after } \\
\text { baseline } \\
\text { assessment }\end{array}$ & $\begin{array}{l}\text { End of } \\
\text { pregnancy } \\
\text { (birth) }\end{array}$ \\
\hline Informed Consent/Assent & & $\sqrt{ }$ & & & \\
\hline $\begin{array}{l}\text { Review Inclusion/Exclusion } \\
\text { Criteria }\end{array}$ & & $\sqrt{ }$ & & & \\
\hline Participant allocation & & & $\sqrt{ }$ & & \\
\hline $\begin{array}{l}\text { Socio-Demographic } \\
\text { data/Characteristics } \\
\text { history }\end{array}$ & Questionnaire & $\sqrt{ }$ & & & \\
\hline $\begin{array}{l}\text { Maternal anthropometrics } \\
\text { Weight gain during study } \\
(\mathrm{kg}) \\
\text { Height }(\mathrm{cm}) \\
\text { Other } \\
\text { Blood pressure }\end{array}$ & $\begin{array}{l}\text { Digital scale } \\
\text { Digital scale } \\
\text { Mercury } \\
\text { sphygmomanometer }\end{array}$ & & $\begin{array}{l}\sqrt{ } \\
\sqrt{ } \\
\sqrt{ }\end{array}$ & $\begin{array}{l}\sqrt{ } \\
\text { J } \\
\text { J }\end{array}$ & \\
\hline $\begin{array}{c}\text { Biochemical Test } \\
\text { Blood glucose } \\
\text { Haemoglobin }\end{array}$ & $\begin{array}{l}\text { Serum sample } \\
\text { Serum sample }\end{array}$ & & $\begin{array}{l}\sqrt{ } \\
\sqrt{ }\end{array}$ & $\begin{array}{l}\sqrt{ } \\
\sqrt{ }\end{array}$ & \\
\hline $\begin{array}{l}\text { Pregnancy outcomes } \\
\text { Birth weight }\end{array}$ & & & & & v \\
\hline $\begin{array}{l}\text { Dietary } \\
\quad \text { Food intake** }\end{array}$ & $\begin{array}{l}\text { Food records online } \\
\text { MDD-W } \\
\text { questionnaire } \\
\text { AEBQ questionnaire }\end{array}$ & & $\begin{array}{l}\sqrt{ } \\
\sqrt{ } \\
\sqrt{ }\end{array}$ & $\sqrt{ }$ & \\
\hline
\end{tabular}

\section{Sample size $\{14\}$}

The researcher will estimate value based on result of the previous intervention study which investigates the maternal factors correlation with neonatal weight birth at PUSKESMAS Garuda in 2010 (Budiman, 2017). Initial sample size for within groups $n(50)$ is computed as a function of power level 1-b (0.90),

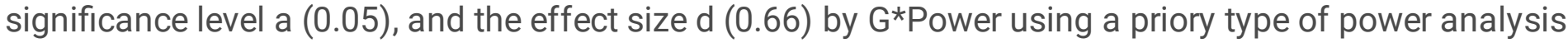
for $t$ test of means different between two independent means (two groups) based on the birth weight 
infant means prediction (control: $2974.0 \pm 417.2 \mathrm{~g}$ and intervention: $3250,0 \pm 417.2 \mathrm{~g}$ ). With assumption drop-out level d.o (10\%), the sample size for within groups $(n *)$ is 56 , making a total of 112 .

\section{Recruitment $\{15\}$}

The recruitment process is carried out at maternal and child health clinics at Community Health centre (PUSKESMAS) and private clinic. Pregnant women will be identified based on arrival and register for regular antenatal care. The principal researcher will approach pregnant women as a recruitment strategy with support from study sites staff. Researchers have access to data information in identifying potential participants through medical records and register books. Besides, the health professional will be informed of the eligibility criteria for potential study participants by the principal researcher. These professionals will be provided with information packs (demography questionnaire and participants' information sheets) to identify pregnant women were meeting the eligibility criteria of the study. In some cases, health professionals review patient records to identify women who are suitable as a subject participant and then informed the principal investigator to be followed up.

Pregnant women who interested in participate the study were asked for their contact details to obtain further information. No personal data was provided to the researcher without the women's consent. During the first stage, pregnant women were asked information about the age, gestational age, and smartphone ability, along with information on any medical condition, with the intention of pre-screening pregnant women who did not meet the inclusion criteria. If the pregnant women did not meet the inclusion and exclusion criteria (e.g., outside the age range and have medical problem), they were not invited for baseline measurements. It was politely explained to them that they not eligible. Those who meet the criteria were obtained (MDD-W) and AEBQ questionnaire.

If investigator found the consent was invalid, there has been a substantial change to the research, or the subject condition since the time of the original consent signed. The investigator will contact the participants in person, by phone, explain the need for re-consent, and give participants an opportunity to ask questions. In addition, investigators ask the participants to sign a document affirming their willingness to continue participate in research study.

\section{Assignment of interventions: allocation}

\section{Sequence generation $\{16 \mathrm{a}\}$}

The selecting control group pregnant women who were similar to the intervention group pregnant women based on three similar characteristics, such as maternal age, income, and education level. To allocate between intervention and control groups the researcher will use free online randomizer "QuickCalcs by GraphPad allow a researcher to specify the number of participants and number of groups, and quickly return a list showing which participant go to which groups (White at al., 2018). The researcher notified all pregnant women that they had the opportunity to access the SISFORNUTRIMIL application; however, the randomizer will choose one of them. The selections (intervention or control) were then written on papers 
and placed in sealed, opaque envelopes, which were kept in a locked drawer. Recruited subjects were assigned a code participant and were permitted to pick one of the available envelopes to determine group selection. Each participant will be categorized based on the participant code according to the recruitment location and the participant's characteristics.

\section{Concealment mechanism \{16b\}}

Each participant will be categorized based on the participant code according to the recruitment location and the participant's characteristics. Then, participants will be allocated to intervention or control groups by a random number generated by the computer if the participants have numbered 4,6,8,10 and etc.

\section{Implementation $\{16 c\}$}

The principle investigator with six persons of data collector will generate the allocation sequence, and will enroll and assign participants to interventions.

\section{Assignment of interventions: Blinding}

\section{Who will be blinded $\{17 a\}$}

The trial participants and data collectors will be none or un-blinded intervention. Which the intervention group will be given a username and password after allocation to access the application, and the control group could not open the application because does not registration.

\section{Procedure for unblinding if needed $\{17 \mathrm{~b}\}$}

This study a 'blind' study which the subject or the Investigator (or both) are unaware which the subject of trial as a user or non-user application.

\section{Data collection and management}

\section{Plans for assessment and collection of outcomes $\{18 \mathrm{a}\}$}

The data were collected at baseline assessment, 12 weeks after intervention and the end of pregnancy. Principal researcher assisted with two or three senior students as a research assistant will collect the data. The participants in both groups will be received the same antenatal care procedure. Health care professionals will conduct clinical assessment measurement in three times: two times in mid pregnancy and one time in the end of pregnancy. First measurement will obtained at 22-26 weeks of gestation as baseline assessment included blood pressure and weight gain measurement, non-fasting blood samples and haemoglobin level measurements, also the questionnaires of dietary diversity survey. The second measurement will collect at 12 weeks after intervention, which the measurements are same with baseline assessment, add with food intake measurement. The maternal eating behaviour will obtain by food intake, gestational weight gain, MDD-W and AEBQ questions. Also, pregnancy experience will be assessed 
by laboratory test. Further, the final measurement such as neonatal birth weight will be conduct at the end of pregnancy period.

\section{Plans to promote participant retention and complete follow-up $\{18 \mathrm{~b}\}$}

The only potential risk, when collecting data, may be that participants feel the process of antenatal care visits takes longer. However, the researcher will ensure that the questions asked to pregnant women will focus on the related information need which is not accessible on their medical record. If the participant is tired, the data collection will be stopped temporally and offered the participant to take a rest.

If in collection data show any type potentially concern about unwanted health problems, the researcher will refer the women to health care professionals.

\section{Data management $\{19\}$}

The data will be stored on Google drive and securely uploaded through a password-protected computer (laptop) to security protected data 'cloud' storage. While food record data will be stored on MySQL database. Where application will store data in MySQL database as structure query language in Entity Relationships Diagram form (ERD). The access password will only be known by researcher and the data will never be left on the computer. Minimal identifiable data were used in this research, and such identifying data have been omitted from this report. Confidentiality was assured at all stages of this research. All data collected will be re-identified and coded to ensure participant anonymity. The information sheet and signed consent forms will be scanned and uploaded to the security protected 'cloud' storage and the original documents will be stored in a password-protected briefcase. The access passwords will only be known by the researcher, who will directly supervise the security protected 'cloud' storage and the briefcase at all times. Only, the research team will have access to secured information.

\section{Confidentiality $\{27\}$}

Confidentiality will be assured at all stages of this research. The information sheet and signed consent forms will be scanned and uploaded to the security-protected 'cloud' storage and the original documents will be stored in a password-protected briefcase. The access password will only be known by the researcher, who will directly supervise the security-protected 'cloud' storage and the briefcase at all times. These data collected and research finding will be presented by code and researcher keep the data collected for five years.

Participant confidentiality will be maintained by anonymity or any identifying personal information on any kind of data collected and measurement instruments. Names of the participants will not be attached to the dataset. Only the participants' medical record number and participants' code will be attached on the document.

Plans for collection, laboratory evaluation and storage of biological specimens for genetic or molecular analysis in this trial/future use $\{33\}$ 
The type of biospecimen in this study is sample blood. According to the procedure, pregnant women will be taken 3cc of blood from venous by laboratory staff, and then staff analyzed the blood to find out the $\mathrm{HB}$ and glucose levels. The results were written in the format and patient medical records.

\section{Statistical methods}

\section{Statistical methods for primary and secondary outcomes $\{20 \mathrm{a}\}$}

Overall, data processing and statistical analysis will be analysed using the SPSS statistical software package version 25. Descriptive statistics will be used to display the study population's baseline characteristics and the mean value of outcome measures. Data will be analysed to determine whether there are any statistically significant differences between the groups. Data will be analysed by using appropriate parametric or non-parametric tests such as two independent sample t-test or Mann u Whitney test, and ANOVA test will use for continuous data dependent on the distribution. Data will be analysed prospectively to examine the relationship between variables and maternal characteristics by using simple linear regression and multiple linear regression. Chi-square test will use for categorical variables. Mean (SD), or median (IQR) will report for continuous data. Number and proportions will report for categorical data. The statistical significance will set to $5 \%$, where a p-value of $<0.05$ is statistically significant.

\section{Interim analyses $\{21 \mathrm{~b}\}$}

There are no organisational data will be used in this research. If there are any obtain personal data from other sources, investigator must provide the individuals with privacy information immediately.

\section{Methods for additional analyses (e.g. subgroup analyses) \{20b\}}

The focus group discussion will be conducted to evaluate feasibility and accessibility of SISFORNUTRIMIL application. The data collection will be conducted in March, 2020 with the SISFORNUTRIMIL user groups. For qualitaive study, I chose thematic analysis and the initial data coding will checked by the supervisors, which helped in enhancing rigour in the analytical process (researcher's triangulation).

\section{Methods in analysis to handle protocol non-adherence and any statistical methods to handle missing data $\{20 \mathrm{c}\}$}

To anticipate the possible reasons for missing data (e.g. patient refusal to continue in the study, patient withdrawals due to treatment failure, treatment success or adverse events, patients moving), the principle investigation will be withdrawn this respondent from this study and replaced by the new one participant. Every effort will be made to anticipate missing data and where appropriate, multiple imputations will be used to handle missing data.

Plans to give access to the full protocol, participant level-data and statistical code $\{31 \mathrm{c}\}$ 
The researchers will identify the pregnant women when they registered at the registration counter of PUSKESMAS. All pregnant women were aged 19 and over will be provided with participant information sheets and they can read while waiting in a queue number to register at a maternal and child clinic. Then, individuals will be screened for eligibility. The eligible participants indicating a desire to participate were first given further details of the study, completed screening questionnaires and information sheet and consent form. They will be determined in the baseline meassurement survey. Participant's name, mobile phone number and contact information will be kept in protected file on secure place. The full protocol will be given for public access no later than 3 years after the collection.

\section{Oversight and monitoring}

\section{Composition of the coordinating centre and trial steering committee $\{5 \mathrm{~d}\}$}

The progress of the trial overll conduct by the group includes the principal investigator and other relevant members such as statistician, database programmer, nutrisionist, six data colector and supervisor from seven site location. The group meetings are essential to keep members up -to-date with the trial and to monitor progress. The frequency of meetings is trial dependent; however, it is recommended that this group would meet frequently during trial set-up and at least quarterly thereafter. A meeting should also be held before a group meeting to plan the agenda and required meeting notes.

\section{Composition of the data monitoring committee, its role and reporting structure $\{21 \mathrm{a}\}$}

Usually the data monitoring commitee is the only group to have access to unblinded data during the course of the trial. For this study, a data monitoring committe is not needed, because the trial using lowrisk procedure, and as such the researcher will be monitoring the data. In addition, it considers whether or not any interim analyses are required and would review these data (applicable to blinded trials only). All research members should be totally independent of the trial.

\section{Adverse event reporting and harms $\{22\}$}

The food record offline will be given to intervention group to anticipate they could not access the application because poorly internet access caused by temporary geography aspect or natural disaster.

\section{Frequency and plans for auditing trial conduct $\{23\}$}

The Trials Auditing will conduct by ethical board staff from the Universitas Padjadjaran, which is responsible for conducting internal audits of applicable clinical trials. Before approval, the full board meeting will be held to review this study.

\section{Plans for communicating important protocol amendments to relevant parties (e.g. trial participants, ethical committees) $\{25\}$}

The changes of protocol modification will be declared in statement letter and communicated to data collectors, ethical commitees and trial registry. 


\section{Dissemination plans $\{31$ a $\}$}

The dissemination of research finding will be held by investigator including the costs of administering. Research reports may be disseminated in debriefing workshops to other investigators, health professionals, and policymakers. There are five key audiences for this research; these are community nursing service provider staff, external statutory organizations (provision health office), obstetricians, nutritionist and academia.

\section{Discussion}

The primary objective of this study is to determine if maternal eating behaviour effects after three months were at standard criteria of Indonesia pregnant women compare with non-users SISFORNUTRIMIL application. The secondary objectives are (1) to determine if clinical and biochemical assessment effects after three months were at an average level, compare with non-user SISFORNUTRIMIL application; (2) to identify the effect of SISFORNUTRIMIL application toward neonatal birth weight, compare with non-user SISFORNUTRIMIL application; and (3) to compare intra-participant effects in primary and secondary objectives for determining whether individual changes were sustained at three months from baseline assessment.

The information system application is expected to assist pregnant women to provide information about the amount of nutrition by suggesting alternatives of types of food that need to be consumed daily (along with their the nutrient description). Therefore, pregnant women can manage their diet and maintain their nutrition intake. Before any evidence-based dietary assessment methods can be effectively adapted and adopted to increase awareness of pregnant women in the improved nutrition quality, the feasibility testing of a mobile technology intervention that influence on maternal eating behaviour and associated maternal is needed.

The comprehensive evaluation of this study will be showed by quantitative analyses and will indicate whether SISFORNUTRIMIL application is a feasible application in complication prevention of pregnant women.

\section{Trial Status}

This study protocol submission date began on 22 October 2019. The recruitment start date: 02 December 2019 and expected recruitment completion date: 30 December 2019. The trial started on 01 January 2020 and end date on 30 April 2020. Version 1.2 approval was obtained from the Universitas Padjadjaran Research Ethics Committee, with a reference letter number: 1227/UN6.KEP/EC/2019 (registration number: 0619060938).

\section{Abbreviations}

SISFORNUTRIMIL: Sistem Informasi Nutrisi Ibu Hamil 
PUSKESMAS: Community Health centre

AEBQ: Adult Eating Behaviour Questionnaire

MDD-W: Minimum Dietary Diversity for Women

\section{Declarations}

\section{Acknowledgements}

We thank our research field, PUSKESMAS kota Bandung and Harapan Keluarga clinic who provided as a field research in this study.

\section{Authors' contributions $\{31 \mathrm{~b}\}$}

Mira Trisyani (MT) conceived and design the research project, also was a major contributor in writing the manuscript. Saseendran Palikadavath (SP) co-writer of the manuscript and supervised the work Mira Trisyani who guided the entire write-up of the manuscript. Isobel Ryder (IR) performed the critical revision of the manuscript. Ngianga Kandala (NK) determined the data analysis for this study

\section{Funding $\{4\}$}

This study will be financial supporting by self-funded of the author.

\section{Availability of data and materials $\{29\}$}

The research team, research site and the University of Portsmouth Data Management will have access to the final trial dataset according to the The University of Portsmouth Data Management policy.

\section{Ethics approval and consent to participate $\{24\}$}

Ethics approval was obtained from the Universitas Padjadjaran Research Ethics Committee, with a reference letter number: 1227/UN6.KEP/EC/2019 (see appendices 1). The information sheet and a copy of signed consent form will be given to participate for keep. Furthermore, all signed consent forms will be securely stored in a password-protected briefcase accessible only by the researcher.

\section{Consent for publication $\{32\}$}

See at appendices 2

\section{Competing interests $\{28\}$}

The authors declare that there are no conflicts of interest regarding the publication of this paper.

\section{Author details}


Mira Trisyani Koeryaman ${ }^{1}$, Saseendran Pallikadavath ${ }^{2}$, Isobel Ryder ${ }^{3}$, Ngianga Kandala ${ }^{4}$

${ }^{1}$ Senior Lecturer, Nursing Faculty of Universitas Padjadjaran and PhD student at School of Health Sciences and Care Professions, University of Portsmouth, U.K.

${ }^{2}$ Professor of Demography and Global Health, School of Health Sciences and Care Professions, University of Portsmouth, U.K.

${ }^{3,4}$ Senior Lecturer, School of Health Sciences and Care Professions, University of Portsmouth, U.K.

\section{Authors' information (optional)}

Not applicable

\section{References}

Bernal AJ, Jirtle RL. Epigenomic disruption: the effects of early developmental exposures. Birth defects research Part A, Clinical and molecular teratology. 2010;88:938-944

Budiman et al. Faktor Ibu yang Berhubungan dengan Berat Badan Bayi Lahir di Puskesmas Garuda.Tahun 2010. Journal kesehatan kartika ISSN : 9772477 D54DD8.

2010. http://www.stikesayani.ac.id/publikasi/e-journal/files/2011/201112/201112-007.pdf.

Committee, O. N. S. D. P., \& Institute, O. M. Nutrition during pregnancy: Weight gain - nutrient supplements. 1990. Retrieved from http://ebookcentral.proquest.com

Chan A, Scheil W., Scott J, Nguyen A., Sage L. Pregnancy outcome in South Australia 2009. Adelaide: Pregnancy outcome unit S.A. Health, Government of South Australia; 2011. Online document.

Committee to Review WIC Food Packages; Food and Nutrition Board; Institute of Medicine; National Academies of Sciences, Engineering, and Medicine; Rasmussen KM, Latulippe ME, Yaktine AL, editors. Review of WIC Food Packages: Proposed Framework for Revisions: Interim Report. Washington (DC): National Academies Press (US); 2016 Jul 6. 6, Nutrition-Related Health Risks in the WIC Population. 2016. Available from: https://www.ncbi.nlm.nih.gov/books/NBK379056/

Chan A-W, Tetzlaff JM, Altman DG, Laupais A, et al. SPIRIT 2013 Statement: Defining standard protocol items for clinical trials. Ann Intern Med. 2013;158(3):200-207.

Dovey T. Eating behaviour. Retrieved from http://ebookcentral.proquest.com

Grosvenor, M.B., Smolin, L.A. (2006). Nutrition: Everyday choices ( $1^{\text {st }}$ ed.) United States: John Wiley \& Sons. Inc; 2010. 
Institute of Medicine. Dietary Reference Intake for Energy, Carbohydrate, Fiber, Fat, Fatty Acids, Cholesterol, Protein, and Amino Acids. A Report of the Panel on Macronutrients, Subcommittees on Upper Reference Levels of Nutrients and Interpretation and Uses of Dietary Reference Intakes, and the Standing Committee on the Scientific Evaluation of Dietary Reference Intakes. Washington, DC: National Academies Press; 2005.

Lee RD, Nieman, DC. Nutritional Assessment, Fifth edition. North America: The McGraw-Hill; 2010.

Pereira, RA, Araujo MC, Lopes TS, Yokoo EM. How many 24-hour recalls or food records are required to estimate usual energy and nutrient intake? Cad Saúde Pública. 2010;26:2101-11.

Scott J, Campbel D, Davies. Mothers and infants In M. Lawrence \& T. Worsley (Ed). Public Health Nutrition From principle to practice. New York: McGraw-Hill; 2007.

Symington EA, Baumgartner J, Malan L., Zandberg L, Ricci C, and Smuts C. Nutrition during pregnancy and early development (NuPED) in urban South Africa: a study protocol for a prospective cohort. BMC Pregnancy and Childbirth. 2018;18(1).

Singh A, Bains K, Kaur H. Relationship of Eating Behaviours with age, anthropometric measurements, and body composition parameters among professional Indian women. ISSN: 0367-0244 (print), 1543-5237 (online) Journal homepage. 2017; http:www.tandfonline.com/loi/gefn20.

White H, Sabarwal S, and de Hoop T. Methodological Briefs Impact Evaluation No. 7. Unicef: Office of Research-Innocenti; 2018.

World Health Organization. 2016. Country Health Profile: Indonesia: Global Health Observatory. http://www.who.int/countries/idn/en/

Weber MA, Schiffrin EL, White WB, Mann S, Lindholm LH, Kenerson JG, Harrap SB. Clinical practice guidelines for the management of hypertension in the community: a statement by the American Society of Hypertension and the International Society of Hypertension. Journal of Clinical Hypertension (Greenwich, Conn.). 2014; 16(1), 14-26. https://doi.org/10.1111/jch.12237

Wang C, Lin L, Su R, Zhu W, et al. Haemoglobin levels during the first trimester of pregnancy are associated with the risk of gestational diabetes mellitus, pre-eclampsia and preterm birth in Chinese women: retrospective study. BMC Pregnancy and Childbirth. 2018; 18:263.

https://doi.org/10.1186/s12884-018-1800-7.

Yuan X, Liu H, Wang L, Zhang S, Zhang C, Leng J et al. Gestational hypertension and chronic hypertension on the risk of diabetes among gestational diabetes women. J Diabetes Complications. 2016; 30(7): 1269-1274

\section{Supplementary Files}


This is a list of supplementary files associated with this preprint. Click to download.

- Appendices1.pdf

- SPIRITChecklist.pdf 